\title{
Pectus Deformities: Is Kirschner Use Sufficient for Sternal Stabilization?
}

\author{
Fuat Sayir $^{\mathrm{a}, \mathrm{d}}$, Abidin Sehitogullari ${ }^{\mathrm{b}}$, Bunyamin Sertogullarindan ${ }^{\mathrm{c}}$, Ufuk Cobanoglu ${ }^{\mathrm{a}}$
}

\begin{abstract}
Background: The results of correxion surgery of the chest wall deformities are pretty good. The aims of surgery are provide cosmetic improvement and eliminate the negative side-effects of on the cardiopulmonary system. We presented our clinic experince in patients who were operated with pectus deformities.
\end{abstract}

Methods: Between 2003 - 2011, total of 41 patients who underwent modified Rawitch technique with pectus excavatum and pectus carinatum were included in this study. Kirschner wire was used for sternal fixation. Kirshner wire was taken between postoperative 12 - 15 days.

Results: No mortality was observed. Recurrence occured in one patient (2.4\%). Operative time lasted between 2.2 - 3.2 hours (mean 1.8 hours). Age, ranged between $4-26$ years, mean age was 14.3 years. Thirteen of patients were pectus carinatum, and 25 of them were pectus excavatum. Three patients had mixed type deformity. There were no major complications. The morbidity rate was $26.8 \%$. Mean hospital stay was 18.3 days (17 - 28 days).

Manuscript accepted for publication April 19, 2012

${ }^{a}$ Department of Thoracic Surgery, Faculty of Medicine, Yuzuncu Yil University, 65100, Van, Turkey

${ }^{\mathrm{b}}$ Department of Thoracic Surgery, Hospital of Medical Training and Education, 65100, Van, Turkey

${ }^{c}$ Department of Chest Disease, Faculty of Medicine, Yuzuncu Yil

University, 65100, Van, Turkey

${ }^{\mathrm{d}}$ Corresponding author: Fuat Sayir. Email: sayirfuat@yahoo.com

doi: $10.4021 / \mathrm{jcs} 46 \mathrm{w}$
Conclusions: Cosmetic and psychological problems in patients with pectus deformities improved with surgical correction. In addition, this operation eliminates the negative effects of cardiopulmonary system. The stabilization of sternum with Kirschner wire is a cheap, effective and uncomplicated.

Keywords: Deformity; Correction; Cosmetic; Chest wall

\section{Introduction}

Pectus excavatum (PE) deformity is the most frequently seen congenital thoracic wall deformity, and seen in approximately every 400 live births [1]. In a great majority of cases with PE deformity, the deformity becomes pronounced in the first year of life; however, depression (collapse) generally increases in severity in adolescence, when the development of the skeletal system is accelerated. On the other hand, pectus carinatum (PC) may not be noticed markedly by inspection until the period of adolescence which is the period of fastened development of skeletal system [2]. Pectus deformities are five times more common in males compared to females, and PE deformity is six times more frequent compared to PC deformity [3]. Pectus deformities are usually asymptomatic. These deformities that appear in childhood and which are markedly pronounced in adolescence result in various psychological, cosmetic, orthopedic, and physiological disorders, in varying degrees, according to the severity of the malformation. Intermediate and severe deformities generally are fixed surgically in childhood with satisfactory results. Some cases provide an opportunity for surgery in adults, with generally good results. Nevertheless, there have been reports stating that the operations performed in the adult population are not as successful as the ones performed in childhood [4, 5]. The current valid treatment methods are surgical correction procedures. The surgical procedure is most commonly performed for cosmetic reasons [6, 7]. Many surgical techniques have been tried to date, and the Nuss technique, which has gained popularity recently, has been combined with the Rawitch technique, which has always been applied until to- 
day. The aim of surgery is to achieve the normal position of the sternum and to provide a normal appearing thorax wall.

\section{Material and Method}

A total of 41 patients who underwent the modified Rawitch technique for PE and PC in our clinic between 2003 and 2011 were included in the study. Seven of patients were women and 32 were men. Mean age was 14.3 years with a range of 4 - 26 years. Thirteen patients had PC, and 25 had PE. Three patients had mix type deformity. Patients were evaluated with the parameters of age, sex, type of deformity, symptoms, accompanying anomalies, surgical procedure applied, family history, period in which the deformity became visually apparent duration of hospital stay, and morbidity by analysis of file records. Routine plane and lateral chest Xrays were obtained for all patients. Patients were consulted by pediatric or adult cardiologists, which is a routine procedure in our clinic. Electrocardiography (ECG) and echocardiography $(\mathrm{ECHO})$ were performed by cardiologists and additional pathologies were diagnosed. Thorax CT's were obtained in patients with advanced deformity and patients who we thought to have high pectus index. In addition, patients who had additional lung disease and patients who had comorbidities diagnosed by chest X-ray had thorax CT as well. Respiratory function tests were performed in patients older than 12 years with dyspnea symptoms. Postoperative long term follow-up of the patients and controls were evaluated by analyzing the outpatient clinic records.

Psychological distress seen in patients due to cosmetic defects, negative effects of the deformity on respiratory function tests, and history of frequently recurring lung infections were effective in decision making for surgery in our clinic. Patients with the described clinical picture were operated on soon after required preoperative tests were undertaken.

In the family history, four patients $(9.75 \%)$ had a positive history of deformity in another family member or descent. In the majority of the patients (27 cases, $71 \%)$ the deformity was noticed in childhood and was marked after 6 - 7 years of age. It was established from examining the history that the deformity was progressively marked in the prepubertal period, especially in cases with PC and had accelerated with puberty. In 10 cases with increased noticeable deformity in puberty, surgical correction was performed in the same period. Three cases with PC were diagnosed in early childhood and they were operated on in the pre-school period. The case with the earliest age at operation was a fouryear-old patient with PC.

Marked complaints of the cases were chest wall shape distortion and psychological complaints in all cases, dyspnea on effort in 17 (41.4\%), palpitations in $11(26.8 \%)$, easy exhaustion in eight cases (19.5\%), and frequent lung infections of more than four a year in five cases (12.1\%). Wheezing increased with effort and physical activity and a clinical picture consistent with asthma was identified in three patients. Chest pain was present in two patients. Scoliosis coincided in three patients. Three of the cases with PC had asymmetrical deformity. In cases with PE, the deformity was symmetrical in 19, and asymmetrical in six cases.

The deformity was identified as severe in eight cases with PE. This was confirmed with thorax CT.

One of the important physical examination findings was the narrowing in the anterior-posterior diameter of the chest wall. Mitral valve prolapsus in two cases with PC and in one case with PE was diagnosed with ECHO, and right axial deviation was found in five cases with ECG. In addition, T negativity in V1-2-3 derivations in two cases, and bradicardia in one case were identified by ECG. Decrease in vital capacity (VC) and FEV1 values (forced expiratory volume in the first second) was found in eight patients (21\%) who had dyspnea on effort and had undergone respiratory function tests (RFT).

The study protocol was carried out in accordance with the Helsinki Declaration as revised in 2000 . The study protocol was approved by the local ethics committee, and informed consent was obtained from parents of participants.

\section{Surgical technique}

Operations were performed under general anesthesia, in the supine position, after single lumen endotracheal intubation. Modified Rawitch procedure was performed in 39 cases and single sided costoplasty in one case. In the Modified Rawitch procedure, which was the most frequently used technique, a longitudinal incision was made on the sternum and it was deepened to xifoid process. Skin flaps were modified sufficiently, lateral and bilateral dissections were continued to the intercostal space until the deformed cartilages were seen. Pectoralis muscles were freed by cauterization and two different techniques. In the first technique, they were lifted up like a flap without damaging the muscle. In the other technique, each perichondrial compartment was freed separately by a dissection performed until the intercostal space was reached. All deformed cartilages were bilaterally resected subperichondrially and with conservation of the periost beds. This procedure was continued until the second costal cartilage and costal arc were reached, including all deformed cartilages. The sternum was freed from the xifoid process and bilateral intercostal structures and bands with dissection. After xifoid resection, bands and adhesions in the retrosternal space were dissected by blunt finger dissection under the sternum. Wedge osteotomy was performed on the sternum. To perform this, a single transverse osteotomy at the posterior angulation of the sternum and the last deformed cartilage which was generally at the level of second or third cartilage and at a place which does not articulate with the sternum was used. The sternum was fractured at this point and brought into a flat position. The osteotomy was primary sutured to al- 
low the sternum to stand straight. Kirschner wire was placed behind the sternum and anterior to the costae after positioning the sternum. Thus, paradox development and sternum stabilization were provided. A hemovac drain was placed in the substernal space. In cases with pleural opening, tube thoracostomy was performed on that side. Perichondriums in the bilateral spaces, intercostal, pectoral and rectus muscles, in an order, and skin and subcutaneous tissues were sutured with appropriate suture material.

The duration of the operations was between 2.2 and 3.2 hours (mean 1.8 hours). No serious blood loss occurred. The cases were followed-up in the intensive care unit postoperatively for one or two days. Intravenously cefazolin sodium was given for prophylaxis for 3 days-1 week. Pain control was provided by anti-inflammatory drugs.

\section{Results}

Retrosternal Kirschner wire was not applied in three cases among all those operated on. It was placed in the rest of the patients. In one case without Kirschner placement, a recurrence $(2.4 \%)$ occurred that was markedly obvious, starting from the sixth post-operative month. This case was re-operated on. Kirschner wire was kept in place for 45 days after the procedure. This case is in its 3rd postoperative year, with no problems. No recurrences were seen in patients with Kirschner wire placement.

Morbidities were seen in a total of 11 cases $(26.8 \%)$. Seroma was the most frequently seen postoperative problem, and was identified in five cases. Other main complications, in order of frequency, were wound infection in four cases, pneumothorax in one case, and effusion in one case. Seroma and wound infections were treated with appropriate antibiotics and drainage. Cases with pneumothorax and effusion were treated by tube thoracostomy. One case had revision surgery due to a marked hypertrophic scar, which became obvious after the third postoperative month after consulting with plastic surgery. Kirschner wires were pulled out on the 13 - 15th days, according to the clinical status of the patients and after observing the wound. It was removed on day 45 after reoperation in the patient with recurrence. Hospital stay of the cases varied between 17 to 28 days (mean 18.3 days). Patients were followed-up after surgery for three months to six years. During the follow-up process, $33(80.4 \%)$ of the patients expressed that they were very much pleased with results; however, three patients $(7.3 \%)$ stated that they were not pleased at all. The other five cases (12.1\%) expressed intermediate satisfaction. Patients, who defined respiratory distress in the preoperative period, defined a better clinical picture after the sixth postoperative month; however, no significant difference was found in the respiratory function tests performed after the sixth month. The preoperatively observed complaints, such as limited tolerance to exercise, fre- quent lung infections, and complaints such as asthma were markedly improved. The heart was seen to get into its normal position in the control chest X-rays. Tachycardia seen preoperatively in the patients was ameliorated, which was verified by physical examination.

\section{Discussion}

Pectus deformities are the most frequently seen congenital thorax deformities. They are seen in one in every $300-400$ live births [3]. A genetic relation has not been identified; however, it is assessed as a congenital deformity since a family history is present in approximately $40 \%$ of the cases with the deformity [8-11]. In four of our patients (9.75\%), a family or descent history of a case with deformity was present. The most widely accepted opinion about the development of the deformity is the anterior or posterior displacement of the sternum due to over growth of the lower costal cartilage and the costae, and probable diaphragm involvement in this displacement [12-14]. Various suggestions about the rate of $\mathrm{PE} / \mathrm{PC}$ have been made. Changing rates as $2 / 1$ to $9 / 1$ are reported $[6,14-17]$. This rate was found to be a little higher in our study $(28 / 13=2.15)$. Pectus deformities affect men more often. This rate is reported to be $4-5 / 1$ in the literature. This rate in our study was identified as $34 / 7=4.85$.

PE deformity can be encountered at birth; however, its most frequently diagnosed in early childhood. Nevertheless, patients share with their physicians that with the rapid growth in the adolescent period and completion of skeletal maturation, the depression in the deformity severely increases. This experience was identified in 27 of our patients. With the increased severity of the deformity in the period of completion of skeletal maturation, cosmetic anxiety may transform into a different and an even worse health problem due to gradually worsening respiratory and cardiac problems. In the history of eight cases with these problems that were operated on in adulthood, it was stated that the deformity had been accelerated in puberty and the complaints had increased in the same period. On the other hand, the PC cases were the ones who presented at an older age with primarily cosmetic concerns in all of them. In 10 of these cases, the deformity increased in severity after puberty.

Although PE deformity is tolerated well in early childhood and in childhood periods, with the increasing age together with the completion of skeletal development, it may constitute an absolute indication of operation in some cases due to respiratory and cardiovascular problems. The most frequently seen complaints are pain, cosmetic deformity, and psychological complaints in children who are generally asymptomatic. Palpitations, lower respiratory infections, and asthmatiform complaints might accompany those symptoms. In cases with severe depression, decreased exercise tolerance and systolic murmur can occur. This is due to the pressure 
of the sternum on the pulmonary artery. The most common finding in older children and young adults is the constriction in respiratory capacity. A marked decrease is seen in $\mathrm{VC}$ and FEV1 in severe cases.

The degree of deformity plays an important role in the identification of the indication of surgery in cases with pectus deformity. Surgical indication is cosmetic in especially young cases with mild deformity with primarily cosmetic concerns; however, surgery is mandatory in patients with severe PE deformity with medical problems (such as dyspnea, frequent lung infections, marked decrease in exercise tolerance, and cardiac problems).

Surgical correction can be performed in cases with pectus deformity safely with minimal risk. Corrective surgery can be performed in young patients with success due to the completion of skeletal maturation [3]. However, the main problem arises in the younger patient group whose skeletal maturation is still incomplete. Currently, most surgeons recommend surgery in adolescence or young adulthood. The recurrence risk is also lower in cases with complete bone growth and development [3]. If there is severe cardiopulmonary pressure in younger children, these cases may as well be operated on earlier. Nevertheless, repair at a very early age might result in inappropriate thoracic wall development, recurrence, and other complications [18-20]. Cases with PE are recommended to be operated on in the early adolescence period in our clinic; however, symptoms and the psychosocial status of the patients are taken into account as well. The symptoms increased and the deformity became more marked in a significant number of our patients (10 cases) with puberty; therefore, these patients were operated on in the peripubertal period. The repair of PC is recommended to be performed in late adolescence or young adulthood since there is a chance of recurrence due to bone growth [21]. Nevertheless, there are reports enouncing no development of recurrence in patients operated on in at an early age [22]. We have not encountered any problems in the follow-up period of five years in a case with PC who was operated on at the age of four.

As an alternative to the open techniques currently performed in the treatment of pectus, a minimally invasive technique, developed by Nuss and modified by Pilegaard (Nuss procedure) is successfully employed in both children and in patients in the adolescence period. However, to date in our clinic, open surgical techniques have been used. In the open technique, it is imperative to resect all deformed costal cartilages and perform sternum correction and sternum osteotomy. The sternum must be separated bilaterally from all deformed cartilages and intercostal bands (all deformed cartilages from the 2 nd intercostal ark to arcus costarium) and must be corrected with wedge osteotomy. Otherwise, recurrence is inevitable. We placed retrosternal Kirschner in all but three cases and thus we think that Kirschner must be applied for stabilization in open surgery. Usage of prolene mesh and other synthetic grafts are reported for the stabilization of the sternum; however, we find it advisable to use Kirschner wire since these products are expensive and difficult to supply. The development of no recurrences in our cases also directed us to this opinion. A recurrence occurred in one case with PE in whom we did not apply Kirschner. We kept the Kirschner in place for a long time after the revision in this case. Reoperation of the case was successful. In cases with single sided cartilage hypertrophy, costal cartilages should most certainly also be excised bilaterally and sternum correction must be performed in addition to sternum osteotomy. Otherwise, the deformity becomes worse than the previous one.

In cases that were operated on because of their cardiopulmonary symptoms in our clinic, the symptoms ameliorated in all patients. An obvious improvement in pulmonary function tests was not identified; however, subjective complaints in all patients were improved.

In conclusion, we suggest that surgery in pectus deformity should be performed in young adulthood as long as no severe cardiopulmonary comorbidities are present. To avoid recurrence and to obtain an ideal result, we believe that sternal osteotomy should be performed in addition to bilateral separation of the sternum from all tissues, and a support placement applied to the posterior of the sternum for stabilization, regardless; and that Kirschner wire is ideal for this purpose.

\section{References}

1. Molik KA, Engum SA, Rescorla FJ, West KW, Scherer LR, Grosfeld JL. Pectus excavatum repair: experience with standard and minimal invasive techniques. J Pediatr Surg. 2001;36(2):324-328.

2. Fonkalsrud EW, Beanes S. Surgical management of pectus carinatum: 30 years' experience. World J Surg. 2001;25(7):898-903.

3. Jaroszewski D, Notrica D, McMahon L, Steidley DE, Deschamps C. Current management of pectus excavatum: a review and update of therapy and treatment recommendations. J Am Board Fam Med. 2010;23(2):230239.

4. Morshuis WJ, Mulder H, Wapperom G, Folgering HT, Assman M, Cox AL, van Lier HJ, et al. Pectus excavatum. A clinical study with long-term postoperative follow-up. Eur J Cardiothorac Surg. 1992;6(6):318-328; discussion 328-319.

5. Lancaster L, McIlhenny J, Rodgers B, Alford B. Radiographic findings after pectus excavatum repair. Pediatr Radiol. 1995;25(6):452-454.

6. Robert C, Shamberger. Chest wall deformities. In: Thomas W.Shields General Thoracic Surgery. Philadelphia: Williams and Wilkins, 1994: 529-57. 
7. Tjan TD, Semik M, Rotering H, Rolf N, Scheld HH. Pectus excavatum: special surgical technique, perioperative management and long-term results. J Cardiovasc Surg (Torino). 1999;40(2):289-297.

8. Sabiston JH Jr. Congenital deformities of the chest wall. In: Sabiston JH, ed. Textbook of Surgery. Philadelphia: WB Sauders Co.; 1997.

9. Shamberger RC. Congenital chest wall deformities. Curr Probl Surg. 1996;33(6):469-542.

10. Waters P, Welch K, Micheli LJ, Shamberger R, Hall JE. Scoliosis in children with pectus excavatum and pectus carinatum. J Pediatr Orthop. 1989;9(5):551-556.

11. Kelly RE, Jr. Pectus excavatum: historical background, clinical picture, preoperative evaluation and criteria for operation. Semin Pediatr Surg. 2008;17(3):181-193.

12. Bentz ML, Futrell JW. Improved chest wall fixation for correction of pectus excavatum. Br J Plast Surg. 1992;45(5):367-370.

13. Bentz ML, Rowe MI, Wiener ES. Improved sternal fixation in the correction of pediatric pectus excavatum. Ann Plast Surg. 1994;32(6):638-641.

14. Fonkalsrud EW, Salman T, Guo W, Gregg JP. Repair of pectus deformities with sternal support. J Thorac Cardiovasc Surg. 1994;107(1):37-42.

15. Genc O, Kamali SD. Surgical treatment of Pectus Exca- vatus Deformity. GATA Bulletin. 1996; 38: 507-512.

16. Haller JA, Jr., Scherer LR, Turner CS, Colombani PM. Evolving management of pectus excavatum based on a single institutional experience of 664 patients. Ann Surg. 1989;209(5):578-582; discussion 582-573.

17. de Matos AC, Bernardo JE, Fernandes LE, Antunes MJ. Surgery of chest wall deformities. Eur J Cardiothorac Surg. 1997;12(3):345-350.

18. Jaroszewski DE, Fonkalsrud E. Pectus deformity in the adult patient: cardiopulmonary symptoms as an indication for repair. Presented at the 27th annual International Scientific Assembly of the American College of Chest Physicians, Salt Lake City, Utah; 2006.

19. Jaroszewski DE, Fonkalsrud EW. Repair of pectus chest deformities in 320 adult patients: 21 year experience. Ann Thorac Surg. 2007;84(2):429-433.

20. Jaroszewski D, Steidley E, Galindo A, Arabia F. Treating heart failure and dyspnea in a 78-year-old man with surgical correction of pectus excavatum. Ann Thorac Surg. 2009;88(3):1008-1010.

21. Pickard LR, Tepas JJ, Shermeta DW, Haller JA, Jr. Pectus carinatum: results of surgical therapy. J Pediatr Surg. 1979;14(3):228-230.

22. Shamberger RC, Welch KJ. Surgical correction of pectus carinatum. J Pediatr Surg. 1987;22(1):48-53. 